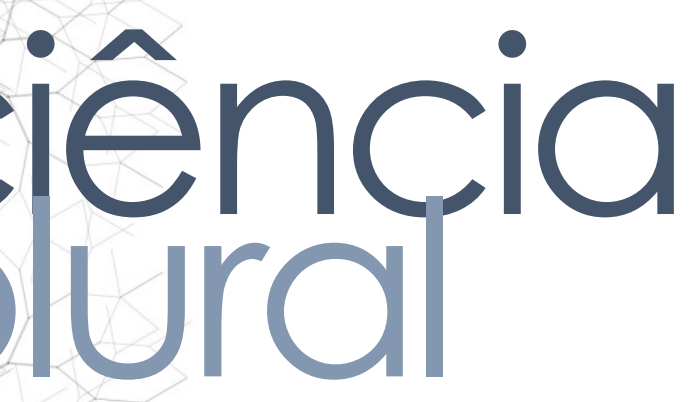

\title{
REABILITAÇÃO ESTÉTICA ANTERIOR COM RESINA COMPOSTA: RELATO DE CASO
}

\section{Anterior esthetic rehabilitation with composite resin: a case report}

Isabela Dantas Torres de Araújo || Universidade Federal do Rio Grande do Norte || Cirurgiã-dentista pela UFRN / Mestranda em Ciências Odontológicas com área de concentração em Clínicas Odontológicas pela UFRN / Especialista em Saúde Coletiva com Ênfase em Saúde da Família pela FACISA/UFRN / Especialista em Dentística pela Academia Norte-RioGrandense de Odontologia/ || E-mail: isabeladta@gmail.com

Priscila Silva Abrantes || Universidade Federal do Rio Grande do Norte | | Cirurgiã-dentista pela UFRN / Mestranda em Ciências Odontológicas com área de concentração em Clínicas Odontológicas pela UFRN / Especialista em Dentística pela Academia Norte-Rio-Grandense de Odontologia || E-mail: priabrantesrn@hotmail.com

Boniek Castillo Dutra Borges || Universidade Federal do Rio Grande do Norte || Doutor em Odontologia com área de concentração em Dentística pela UPE / Professor Adjunto do Departamento de Odontologia da UFRN / Mestre em Clínica Odontológica com área de concentração em Dentística pela UNICAMP / Especialista em Dentística pelo CFO || E-mail: boniek.castillo@gmail.com

Isauremi Vieira de Assunção || Universidade Federal do Rio Grande do Norte || Doutora em Ciências da Saúde pela UFRN / Professora Adjunta IV do Departamento de Odontologia da UFRN / Mestre em Odontologia Social pela UFRN | | E-mail: isauremi@gmail.com

Autor responsável pela correspondência

Isabela Dantas Torres de Araújo || E-mail: isabeladta@gmail.com 


\section{RESUMO}

Introdução: A avaliação do sorriso deve abranger diversos aspectos desde a composição facial até características morfológicas dos elementos dentários e, dessa forma, se qualquer um desses aspectos forem afetados, o sorriso se torna esteticamente inadequado. Objetivo: apresentar um caso clínico cujo plano de tratamento foi composto pela confecção de facetas diretas em resina composta dos elementos antero-superiores a fim de devolver a estética adequada para a paciente. Descrição do caso: Paciente, 16 anos, do sexo feminino, apresentava má oclusão (Classe III de Angle) compensada ortodonticamente, agenesia de incisivos laterais e inversão dental dos elementos 23 e 24. A resolução estética deste caso utilizou a técnica direta com resina composta por meio do protocolo correto no quesito proporção áurea e mimetização. Conclusão: As restaurações diretas em resina composta são uma solução simples, efetiva e prática na intervenção de desarmonias de forma e tamanho dental e, portanto, foi possível reanatomizar os dentes anteriores devolvendo estética, função e satisfação ao paciente.

Palavras-Chave: Estética dentária. Facetas Dentárias. Resinas Compostas.

\section{ABSTRACT}

Introduction: Smile evaluation should cover several aspects from facial composition to morphological characteristics of dental elements and, therefore, if any of these aspects are affected, the smile becomes aesthetically inadequate. Objective: to present a clinical case whose treatment plan was composed by the making of dental veneers in composite resins of the upper and lower elements in order to obtain a suitable aesthetic. Case description: A 16-year-old female patient presents malocclusion (Class III of Angle) orthodontically compensated, agenesis of lateral incisors and dental inversion of elements 23 and 24 . The aesthetic resolution of this case used the direct technique with composite resin by means of the correct protocol in relation to golden proportion and mimicry. Conclusion: Direct restorations in composite resin are a simple, effective and practical solution in the intervention of disharmonies of form and dental size and, therefore, it was possible to reanatomize the anterior teeth restoring aesthetics, function and patient satisfaction.

eywords: Esthetics. Dental Veneers. Composite Resins. 


\section{Introdução}

A avaliação estética do sorriso deve compreender diversos aspectos desde a composição facial até características morfológicas dos dentes. Em todo o caso, se qualquer um desses aspectos forem afetados, o sorriso torna-se esteticamente inadequado como no caso clínico deste estudo que identifica-se a agenesia de incisivos laterais, inversão de posição de elementos, e deficiência maxilar devido à má oclusão Classe III de Angle, que foi compensada ortodonticamente ${ }^{1,2}$.

As agenesias dentárias são classificadas como anomalia dentária de número cuja etiologia é multifatorial. São relativamente frequentes na dentição permanente apresentando uma prevalência que varia entre 0,03 e 10,1\% ${ }^{3}$. O elemento dentário que mais se encontra ausente é o $2^{\circ}$ pré-molar inferior, seguido do incisivo lateral superior, este último sendo o caso da paciente deste caso clínico ${ }^{3}$. Ainda, a presença desta anomalia dentária na região anterior da boca mostra-se como um dos fatores que afetam negativamente a harmonia do sorriso. Diante disto, o tratamento restaurador adesivo direto se torna um método simples, efetivo e minimamente invasivo uma vez que apresenta reversibilidade e tempo reduzido de tratamento principalmente em pacientes que ainda não completaram o crescimento ósseo total ${ }^{2,3,4}$.

Com relação às más oclusões, representam desvios de normalidade das arcadas dentárias, do esqueleto facial ou de ambos, com variações negativas na função, aparência e autoestima dos indivíduos afetados. São o terceiro problema de saúde bucal no mundo, precedidas pela cárie dentária e doença periodontal ${ }^{5}$. Verifica-se que a má oclusão é mais frequente no sexo feminino e em crianças com oito e nove anos e, a má oclusão de Classe III mostra-se menos frequente ${ }^{5}$.

Alguns procedimentos são sugeridos para devolver a estética como, por emplo, clareamento, microabrasão, confecção de facetas diretas e indiretas 2,6 , do a restauração adesiva direta, uma solução simples, efetiva e prática na ervenção de desarmonias de forma e tamanho dental por meio de resinas 
compostas adequadas para esmalte e dentina. Este procedimento caracteriza-se pela conservação de estrutura dental, acréscimo ou diminuição do material, menor tempo e reversibilidade do tratamento 2,7 . Além disso, princípios básicos como cor, forma, tamanho, textura e brilho são essenciais para a formação de um sorriso harmônico principalmente quando utilizados em dentes anteriores ${ }^{2}$.

Com a exigência estética em constante crescimento, os materiais restauradores vêm obtendo novas características a fim de mimetizar a estrutura dental com maiores detalhes ${ }^{2,4,7}$. As resinas compostas de última geração como as nanoparticuladas e nanohíbridas viabilizam o resgate das propriedades de opalescência e fluorescência dos dentes restaurados. Além das cores desse material, as resinas compostas podem conter tons de opacidade ${ }^{6}$.

Visto essa complexidade dos materiais restauradores, é importante que o cirurgião-dentista compreenda os princípios dos sistemas adesivos, das resinas compostas, da técnica a ser utilizada e, não menos importante, ter uma visão multidisciplinar para diagnosticar e planejar o caso corretamente, a fim de garantir o sucesso funcional e estético do tratamento $2,6,7$.

Diante desse contexto, este trabalho objetivou apresentar, mediante um relato de caso clínico, a resolução estética e funcional dentária, por meio do uso de resina composta direta, de uma paciente que apresenta Classe III de Angle compensada ortodonticamente, agenesia de incisivos laterais e inversão dental do elemento $23 \mathrm{em} 24$. Optou-se por esse tratamento devido a fatores como idade do paciente, gerar menor tempo clínico, menor desgaste de tecido dental sadio e manter espaço para os futuros implantes que serão inseridos quando a paciente completar os 18 anos de idade. 


\section{ciênciucia
purl}

\section{Relato de Caso}

Paciente S.A.M.R., 16 anos, sexo feminino, foi encaminhada ao curso de Especialização em Dentística da Universidade Federal do Rio Grande do Norte (UFRN) por meio do Curso de Especialização em Ortodontia da Associação Brasileira de Odontologia do Rio Grande do Norte (ABO-RN) para realizar procedimento estético dos elementos anteriores devido ao formato dos dentes.

Clinicamente, a paciente apresentava dentes hígidos, alinhados, agenesia dos elementos 12 e 22 e inversão dos elementos 23 e 24 (Figura 1). Para a resolução das agenesias, provisórios foram confeccionados com dentes de estoque, fixados ao fio ortodôntico durante atendimento no Curso de Especialização em Ortodontia e, após completar 18 anos de idade, serão realizados implantes dentais. Nos elementos 13, 11 e 21 foram confeccionadas facetas diretas em resina composta e reanatomização do 23 em 24 e 24 em 23 com o intuito de aumentar volume, altura e largura e, por conseguinte, trazer harmonia ao sorriso aplicando-se as normas de proporção estética e proporção dourada considerando que a paciente apresentava espaços iguais $(8 \mathrm{~mm})$ em cada incisivo superior.

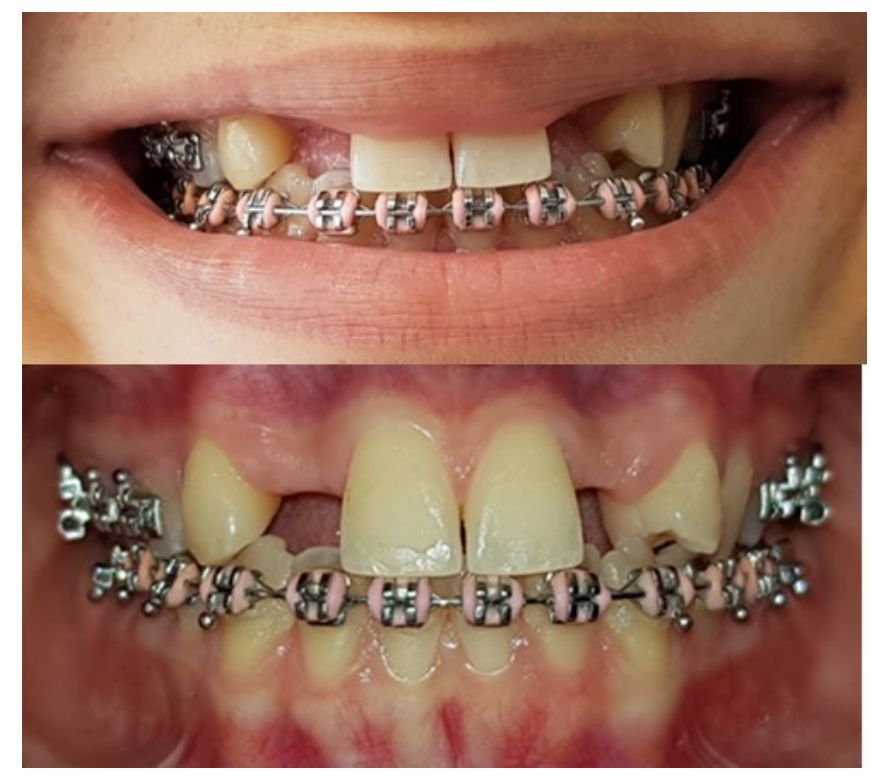

Figura 1. Aspecto clínico inicial. Natal-RN, 2018. 
De acordo com a avaliação ortodôntica, a posição dos elementos 24 e 23 foi planejada dessa forma para prosseguir com a reanatomização. O tratamento será retomado e concluído após a colocação dos implantes.

Durante a manipulação mandibular, observou-se que a paciente não possuía guia de desoclusão incisal satisfatória. $\mathrm{O}$ primeiro procedimento realizado foi uma técnica com o intuito de guiar a desoclusão posterior por meio da inserção de resina composta no terço médio-incisal da palatina dos elementos anteriores superiores.

O esmalte dental do terço médio-incisal da palatina dos elementos 11 e 21 foi condicionado com ácido fosfórico Condac 37\% (FGM, Joinville, SC, Brasil) durante 30 segundos, seguido de lavagem abundante com spray de ar/água. Com o substrato seco, foram aplicadas duas camadas consecutivas do adesivo Prime \& Bond 2.1 (Dentsply, Petrópolis, RJ, Brasil) seguido de fotoativação com o fotopolimerizador Valo Cordless (Ultradent, Indaiatuba, SP, Brasil) por 20 segundos, que é o tempo preconizado pelo fabricante do aparelho. Incrementos de resina composta nanohíbrida IPS Empress Direct (Ivoclar Vivadent, Barueri, SP, Brasil) na cor DA3 foram inseridos e fotopolimerizados durante 20 segundos seguido da checagem da oclusão utilizando um filme de carbono Arti-Fol Metallic 12 micras Dupla-face BK-28 (Bausch, Itu, SP, Brasil).

Durante a segunda sessão uma simulação dos elementos 13, 11 e 21 foi realizada com resina composta nanohíbrida IPS Empress Direct (Ivoclar Vivadent, Barueri, SP, Brasil) nas cores DA3 e EA3 a fim de se avaliar a proporção e cor das facetas com posterior aprovação da paciente.

Ainda, o isolamento absoluto modificado foi utilizado seguido da asperização dos elementos a serem reanatomizados com pontas diamantadas 2135 e 1190 (KG Sorensen, Cotia, SP, Brasil) em conjunto com a mesma sequência condicionamento ácido, adesivo e inserção da resina para os elementos 11 e (Figura 2A) e, em seguida, foi realizado o acabamento e polimento utilizando- 
se lâmina de bisturi $\mathrm{n}^{\mathrm{o}} 12$ para remover o excesso grosseiro do terço cervical e discos Sof-Lex Pop-On (3M ESPE, Ribeirão Preto, SP, Brasil) (Figura 2B).

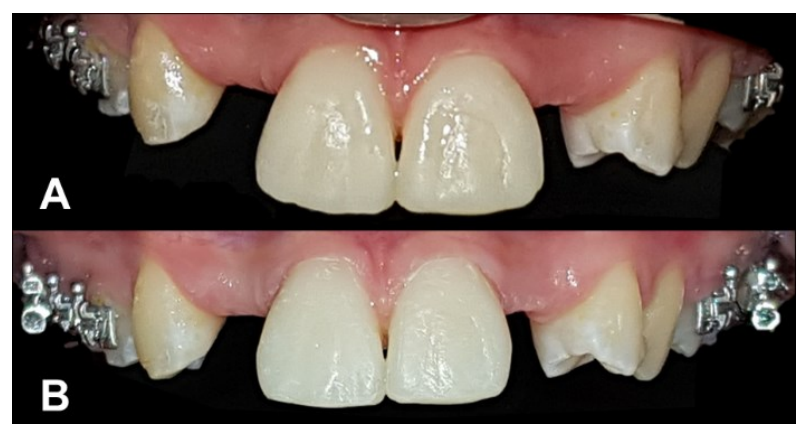

Figura 2. A) Aspecto imediato pós restauração dos elementos 11 e 21. B) Aspecto pós acabamento e polimento. Natal-RN, 2018.

$\mathrm{Na}$ terceira sessão, os mesmos procedimentos de isolamento e sequencia de tratamento de superfície foram realizados para o elemento 13 e para a reanatomização do $24 \mathrm{em} \mathrm{23}$, assim como, para o processo de acabamento e polimento (Figura 3). Por fim, a Figura 4 mostra o caso clínico inicial e final pós polimento.

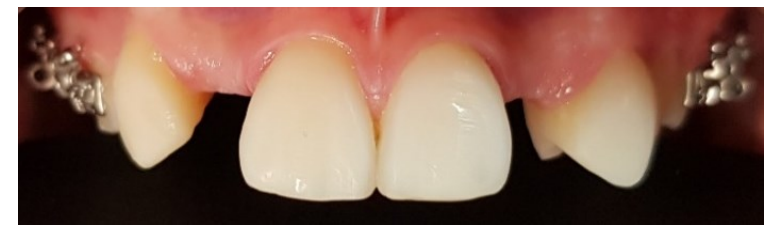

Figura 3. Aspecto imediato pós restauração do elemento $13 \mathrm{e}$ reanatomização do $24 \mathrm{em}$ 23. Natal-RN, 2018.

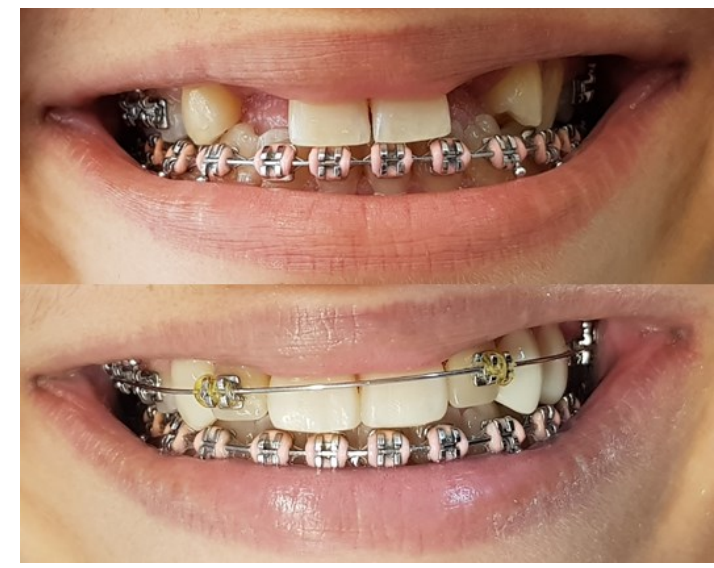

Figura 4. Caso clínico inicial e final das restaurações com provisórios. Natal-RN, 2018. 


\section{Discussão}

Reabilitações estéticas em odontologia têm se tornado rotina em virtude do aumento do interesse dos pacientes por procedimentos que afetam a imagem, vaidade e aceitação social ${ }^{8}$. Dessa forma, durante os últimos 20 anos, houve um avanço tecnológico na odontologia com relação às resinas compostas que permitiu a evolução de suas propriedades físicas, mecânicas e potencial de ligação à substratos por meio dos sistemas adesivos. Além disso, melhor composição e percepção de cores tem sido desenvolvidas ${ }^{6}$.

Esse avanço permite também que os profissionais lancem mão do uso direto desses materiais, possibilitando procedimentos mais conservadores, uma vez que há redução ou eliminação da necessidade de desgastes e preparos cavitários, permitindo devolver aos dentes comprometidos esteticamente, a harmonia de forma e função $2,4,7,9$.

Neste caso clínico, ao empregar a técnica conservadora para a reanatomização dos dentes com resina composta, tornou-se imprescindível aplicar princípios de proporção áurea, proporção estética e propriedades ópticas como opalescência e translucidez ${ }^{2,4,7}$. Embora sejam técnicas comuns, frequentemente essas etapas são negligenciadas, afetando negativamente o resultado final do tratamento provocando insatisfação do paciente.

Dessa forma, a anamnese e exame clínico detalhados tornam-se indispensáveis para o planejamento do tratamento ${ }^{2,7}$. Outrossim, atualmente as resinas compostas possuem grande variedade de cores e efeitos, que favorece combinações variadas de translucidez e opacidade².

A área da estética dentária é muito ampla, envolve princípios científicos e artísticos e está diretamente associada ao sorriso, à harmonia dental, gengiva, ábios e à face como um todo ${ }^{6,11}$. A quebra da ligação entre esses componentes de fomentar problemas no convívio social e de autoestima, conforme a 
realidade e a necessidade de cada paciente $6,7,10,11$. Os valores estéticos são substanciais para a satisfação do paciente e do profissional. Portanto, é elementar que o cirurgião-dentista possua domínio sobre as propriedades físicas e mecânicas das resinas compostas, com a finalidade de articular com a excelência estética da técnica e, por conseguinte, alcançar a tão ansiada harmonia do sorriso ${ }^{7}$.

A correta indicação de facetas diretas com resina composta propicia um tratamento bastante satisfatório, apresentando vantagens de preservação da estrutura dental e simplicidade de técnica10,12-14. Os aspectos que devem ser avaliados com o intuito de confirmar essa indicação são: quantidade e qualidade da estrutura dental remanescente, grau de descoloração, relação entre a área comprometida e distâncias biológicas, análise da oclusão e o grau de higienização do paciente ${ }^{4,9}$. Porém, deve-se atentar a alguns dos fatores que limitam sua indicação, como oclusão topo a topo, ausência de esmalte na região cervical, hábitos parafuncionais e presença de apinhamento dental severo.

Ainda no que tange à oclusão, como visto no caso clínico deste estudo, torna-se claro a necessidade de observar e planejar o caso devidamente, verificando os contatos oclusais existentes em máxima intercuspidação habitual e nos movimentos excursivos de lateralidade e protrusão a fim de se identificar alterações que podem causar falhas nas reabilitações orais. É importante também, o selamento da dentina exposta e restabelecimento da função e estética em casos de fraturas do esmalte e da dentina $2,6,7,9$.

Dentre as vantagens da resina composta, evidencia-se maior preservação dental, menor tempo clínico, baixo custo, boa lisura superficial, facilidade de olimento, radiopacidade, coeficiente de expansão térmica linear relativamente imilar ao da estrutura dental, resistência à compressão, durabilidade, rapidez obtenção dos resultados e reversibilidade ${ }^{12-17}$. Por outro lado, as desvantagens a contração de polimerização que pode ocasionar trincas e infiltração 
marginal quando a técnica não é realizada corretamente e, a baixa estabilidade de cor (manchamento superficial e descoloração interna $)^{7,9,13}$.

O acabamento e polimento realizados de forma correta, proporciona uma superfície lisa e polida que melhora o contorno da restauração, fornece uma anatomia adequada, aumentando a qualidade e a longevidade das restaurações com resina composta $2,10,16,17$.

Principalmente em pacientes jovens, a obtenção do contorno, forma, textura da superfície e mimetização da estrutura dentária é um grande desafio clínico, uma vez que os dentes dessa população específica possuem grande quantidade de detalhes anatômicos ${ }^{18}$. Nestes casos, o esmalte dental é mais branco, possui alta opalescência e baixa translucidez e, a dentina dispõe de um formato de lóbulos bem desenvolvidos e evidentes. A borda incisal apresenta alta translucidez com um fino halo opaco ${ }^{19}$.

Portanto, no presente caso clínico, a paciente não apresentava um sorriso compatível com sua idade e, por meio da técnica direta, foi possível devolver a harmonia almejada utilizando proporções e mimetização adequadas.

\section{Conclusões}

A utilização de resina composta para a reanatomização de dentes anteriores é uma excelente opção quando os princípios estéticos são respeitados, uma vez que possibilita a mimetização da forma, tamanho e cor dos dentes, o que trouxe ao paciente um sorriso harmônico e sua satisfação alcançada. Portanto, o planejamento clínico multiprofissional e em conjunto com o paciente imprescindível para a obtenção do sucesso na reabilitação oral. 


\section{Referências}

1. Mondelli J, Pereira M., Mondelli RF. Etiologia e tratamento dos diastemas dentários. Biodonto. 2003;3(1):11-111. Disponível em: http://bdpi.usp.br/item/001346045

2. Goyatá FDR, Costa HV, Marques LHG, Barreiros ID, Lanza CRM, Novaes Júnior JB, et al. Remodelação estética do sorriso com resina composta e clareamento dental em paciente jovem: relato de caso. Arch Heal Investig. 2017;6(9):408-13. Disponível em:

http:/ / www.archhealthinvestigation.com.br/ArcHI/article/view/2224

3. Cury SEV, Paula JR, Santos PA, Cury MDPN. Hipodontia de dentes permanentes: prevalência e distribuição numa população brasileira. Cad UniFOA. 2015;(29):137-47. Disponível em: http:/ / revistas.unifoa.edu.br/index.php/cadernos/article/view/411/403

4. Sinhori BS, Stolf SC, Andrada MAC. Reanatomização estética de caninos em caso de agenesia de incisivos laterais. Int J Brazilian Dent. 2016;12(1):58-64. Disponível em:

https://www.researchgate.net/publication/306077672_Reanatomizacao_Esteti ca_de_Caninos_em_Caso_de_Agenesia_de_Incisivos_Laterais

5. Sousa JP, Sousa SA. Prevalência de má oclusão em escolares de 7 a 9 anos de idade do Polo 1 da Rede Municipal de Ensino em João Pessoa-PB. Rev Odontol da UNESP. 2013;42(2):117-23. Disponível em:

http:/ / www.scielo.br/scielo.php?pid=S1807-

25772013000200009\&script=sci_abstract\&tlng=pt

6. Silva GR, Fracalossi C, Lucena MTL, Mantovani MB. Tratamento estético com diretas de resina composta - relato de caso. Rev UNINGÁ Rev. 2015;24(3):27-31. Disponível em:

http://revista.uninga.br/index.php/uningareviews/article/view/1724

Rodrigue SDR, Argolo S, Cavalcanti AN. Reanatomização dental com sina composta. Rev Bahiana Odontol. 2014;5(3):182-92. Disponível em: tps://www5.bahiana.edu.br/index.php/odontologia/article/viewFile/565/

420 
8. Wang C, Qin M, Guan Y. Analysis of pulp prognosis in 603 permanent teeth with uncomplicated crown fracture with or without luxation. Dent Traumatol. 2014. Disponível em:

https://www.ncbi.nlm.nih.gov/pubmed/24571370

9. Sanketh AK, Sridevi J, Kalavathy N, Premnath K. Changing smiles with porcelain veneers: a case report. Indian J Dent Sci. 2014;6(3):59-61. Disponível em: http://archieve.ijds.in/issue-pdf-Vol_6_Issue_3_September_2014-26.pdf

10. Pereira DA, Borges MG, Silva FP, Menezes MS. Reabilitação estética do sorriso por meio de procedimento restaurador direto com resina composta nanoparticulada: relato de caso. Rev Odontológica do Bras. 2016;25(72):54-8. Disponível em:

http://www.robrac.org.br/seer/index.php/ROBRAC/article/view/961

11. Lima RBW, Leite JT, França RM, Brito MCT, Uchôa RC, Andrade AKM. Reabilitação estética anterior pela técnica do facetamento - relato de caso. Rev Bras Ciências da Saúde. 2013;17(4):363-70. Disponível em:

http://www.periodicos.ufpb.br/ojs/index.php/rbcs/article/view/13389

12. Frese C, Schiller P, Staehle HJ, Wolff D. Recontouring teeth and closing diastemas with direct composite buildups: a 5-year follow-up. J Dent.

2013;41(11):979-85. Disponível em:

https://www.ncbi.nlm.nih.gov/pubmed/23954577

13. Gomes G, Perdigão J. Prefabricated composite resin veneers - a clinical review. J Esthet Restor Dent. 2014;26(5):302-13. Disponível em:

https://www.ncbi.nlm.nih.gov/pubmed/24942614

14. Soares PV, Spini PHR, Carvalho VF, Souza PG, Gonzaga RCQ, Tolentino $\mathrm{AB}$, et al. Esthetic rehabilitation with laminated ceramic veneers reinforced by lithium disilicate. Quintessence Int. 2014;45(2):129-33. Disponível em:

https://www.ncbi.nlm.nih.gov/pubmed/24389565

Azevedo N, Galvão G, Nihi VSC, Hoeppner MG, Nihi FM. Otimização sorriso com restaurações diretas de compósito resinoso nanoparticulado. VOPAR Cient Ciênc Biol Saúde. 2015;17:43-49. Disponível em: 
http://www.pgsskroton.com.br/seer/index.php/JHealthSci/article/viewFile/ $330 / 309$

16. Can Say E, Yurdagüven H, Yaman BC, Özer F. Surface roughness and morphology of resin composites polished with two-step polishing systems. Dent Mater J. 2014;33(3):332-42. Disponível em: https://www.ncbi.nlm.nih.gov/pubmed/24598241

17. Güngör HC. Management of crown-related fractures in children: an update review. Dent Traumatol. 2014;30(2):88-99. Disponível em: https://www.researchgate.net/publication/257752037_Management_of_crow n-related_fractures_in_children_An_update_review

18. Wittmann K, Giacomelli Neto R, Longo RE, Sene F. Restauração estética do sorriso por meio da integração de técnicas de clareamento e restauração direta de resina composta. Clín Int J Bra Dent. 2013;9:456-467. Disponível em: http://pesquisa.bvsalud.org/portal/resource/pt/lil-786189

19. Sene F, Barbosa K, Vessoni A. Esthetic remodeling of maxillary incisors with composite resin: color, shape and proportion correction. J Clin Dent Res. 2016;13(2):70-7. Disponível em: http://www.dentalpresspub.com/br/jcdr/v13n2/70

Submetido: 10/09/2018

Aprovado: 30/04/2019 